\title{
Prognostic values, ceRNA network, and immune regulation function of SDPR in KRAS-mutant lung cancer
}

\author{
Xiaoqing Luo ${ }^{\dagger}$, Shunli Peng ${ }^{\dagger}$, Sijie Ding, Qin Zeng, Rong Wang, Yueyun Ma, ShiYu Chen, Yanxia Wang \\ and Wei Wang ${ }^{*}$ (D)
}

\begin{abstract}
Background: Serum Deprivation Protein Response (SDPR) plays an important role in formation of pulmonary alveoli. However, the functions and values of SDPR in lung cancer remain unknown. We explored prognostic value, expression pattern, and biological function of SDPR in non-small cell lung cancer (NSCLC) and KRAS-mutant lung cancers.

Methods: SDPR expression was evaluated by quantitative real-time PCR (RT-qPCR), immunohistochemistry (IHC), and Western blot on human NSCLC cells, lung adenocarcinoma tissue array, KRAS-mutant transgenic mice, TCGA and GEO datasets. Prognostic values of SDPR were evaluated by Kaplan-Meier and Cox regression analysis. Bioinformatics implications of SDPR including SDPR-combined transcription factors (TFs) and microRNAs were predicted. In addition, correlations between SDPR, immune checkpoint molecules, and tumor infiltration models were illustrated.

Results: SDPR expression was downregulated in tumor cells and tissues. Low SDPR expression was an independent factor that correlated with shorter overall survival of patients both in lung cancer and KRAS-mutant subgroups. Meanwhile, ceRNA network was constructed to clarify the regulatory and biological functions of SDPR. Negative correlations were found between SDPR and immune checkpoint molecules (PD-L1, TNFRSF18, TNFRSF9, and TDO2). Moreover, diversity immune infiltration models were observed in NSCLC with different SDPR expression and copy number variation (CNV) patterns.
\end{abstract}

Conclusions: This study elucidated regulation network of SDPR in KRAS-mutant NSCLC, and it illustrated correlations between low SDPR expression and suppressed immune system, unfolding a prognostic factor and potential target for the treatment of lung cancer, especially for KRAS-mutant NSCLC.

Keywords: SDPR (CAVIN2), KRAS mutation, Lung cancer, PD-L1, Immunotherapy

\section{Background}

Lung cancer is the most common and lethal cancer among all cancer types [1]. With the conception of individualized therapy [2], significant progress has been made based on specific pathologic subtype and molecular aberrations (e.g., epidermal growth factor receptor

*Correspondence: wangwei9500@hotmail.com

${ }^{+}$Xiaoqing Luo and Shunli Peng contribute equally to this study Department of Radiation Oncology, Nanfang Hospital, Southern Medical University, Guangzhou 510515, People's Republic of China
[EGFR], anaplastic lymphoma kinase [ALK] [3]. Kirsten rat sarcoma viral oncogene homolog $(K R A S)$ mutation is frequently detected in lung adenocarcinoma and closely related with smoking status [4-6]. Several researches show that KRAS mutation is the most common genetic alteration type, and it occurs in approximately 10-25\% of lung cancer in Western and Asia countries [7-9]. The effective clinical strategies of EGFR [10], ALK [11], and rearranged during transfection [RET] [12] aberrations remains to be explored for tumors with KRAS mutations

(c) The Author(s) 2021. This article is licensed under a Creative Commons Attribution 4.0 International License, which permits use, sharing, adaptation, distribution and reproduction in any medium or format, as long as you give appropriate credit to the original author(s) and the source, provide a link to the Creative Commons licence, and indicate if changes were made. The images or other third party material in this article are included in the article's Creative Commons licence, unless indicated otherwise in a credit line to the material. If material is not included in the article's Creative Commons licence and your intended use is not permitted by statutory regulation or exceeds the permitted use, you will need to obtain permission directly from the copyright holder. To view a copy of this licence, visit http://creativeco mmons.org/licenses/by/4.0/. The Creative Commons Public Domain Dedication waiver (http://creativecommons.org/publicdomain/ zero/1.0/) applies to the data made available in this article, unless otherwise stated in a credit line to the data. 
[2]. The $R A S$ gene family encodes a small hydrolyzed guanosine triphosphate GTPase membrane-bound protein, which interacts with downstream effectors to activate transduction of cellular survival signals, such as RAF-MEK-ERK, PI3K-AKT-mTOR, and RALGDSRA [13-15]. Frequent mutant RAS subtypes include KRAS (86\%), neuroblastoma rat sarcoma viral oncogene homolog (NRAS) (11\%), and Harvey rat sarcoma viral oncogene homolog (HRAS) (3\%) [16]. In case of NSCLC, KRAS mutations occur predominantly in codons 12 and 13 , and most frequent variants include $\mathrm{G} 12 \mathrm{C}, \mathrm{G} 12 \mathrm{~V}$, and G12D $[9,17]$.

Recently, a series of compounds targeting KRASG12C variant have been developed and achieved promising effects in preclinical experiments and phase I clinical trials [18-20]. However, it is not clear whether KRAS mutation, especially G12V and G12D variant, can have any clinical benefits. Meanwhile, patients with co-occurring TP53/KRAS mutations showed remarkable clinical response to immune checkpoint inhibitors (CPI) [21]. Moreover, patients with KRAS mutation had favorable clinical benefits of anti-PD-1/PD-L1 immunotherapy [22], and high PD-L1 expression in tumor cells was associated with improved overall survival in KRAS mutant patients [23]. However, the loss of STK11/LKB1 promoted programmed PD-1/PD-L1 inhibitor resistance [24]. These studies indicated that the presence of co-occurring genetic events and the mutant KRAS allelic content increase biological heterogeneities of KRASmutant NSCLC, which complicates the treatment of KRAS-mutant lung cancers.

To investigate the gene expression signature in KRASoncogene-driven lung cancer, we compared the differences between KRAS-mutant tumors and normal lung tissue derived from a genetically engineered mouse model (GEMM), based on expression profiling and comprehensive bioinformatics analysis. Several differentially expressed genes (DEG) were screened according to the gene expression profile, but SDPR was the only DEG that decreased in both GEMM tumors.

SDPR (also known as CAVIN2, NC_000002.12, gene ID 8436), a key substrate for protein kinase $C$, was found to play a critical role in inducing membrane curvature and participating in the formation of caveolae [25]. It has been reported that SDPR is a potential diagnostic indicator in cancers such as hepatocellular carcinoma and gastric cancer [26-28]. However, it remains unknown whether SDPR could be a predictor or target for lung cancer, especially in KRAS-mutant group. Moreover, SDPR is considered a suppressor gene in papillary thyroid cancer [29], but the regulatory mechanism of SDPR remains to be illustrated. Meanwhile, the connection between SDPR and tumor microenvironment (TME) has rarely been explored. Our study explored the gene signature, regulation, and effect of SDPR on tumor and immune infiltration, based on comprehensive bioinformatics analysis, evaluation of lung cancer specimens, and preclinical experiments.

\section{Methods \\ Cell lines and reagents}

Human non-small cell lung cancer cells (HCC4006, H23, H358, SK-LU-1 and H1299) were purchased from American Type Culture Collection (ATCC), Virginia., America. Human embryonal lung cell (MRC-5) was purchased from the Type Culture Collection of the Chinese Academy of Sciences, Shanghai, China [30]. HCC4006, H23, H358 and H1299 cells were maintained in RPMI 1640 supplemented medium, MRC-5 cells were maintained in Dulbecco's Modified Eagle's Medium (DMEM), and SK-LU-1 cell lines was maintained in Minimum Essential Medium (MEM), respectively. All cells were cultured in standard environment as descried previously [31].

\section{Transgenic mouse and establishment of KRAS-mutant lung cancer models}

The LSL-KRAS mice (B6.129S4-KRAStm4Tyj/JNju) were purchased from Nanjing biomedical institution of Nanjing University, Nanjing, China, and housed in specific pathogen-free (SPF) institution of Experimental animal center in Southern Medical University, Guangzhou, China. Cre recombinase induced Adeno-associated viruses (AAV-CMV-bGloin-Cre) were purchase from Shanghai genechem Co., Ltd., China. AAV-CMV-bGloinCre virus was in tracheally instilled into LSL-KRAS mice to induce $K R A S$-oncogene expression [32]. After further 4-6 months, visible tumor nodules were observed in lung tissue. Finally, tumor-bearing mice were sacrificed, and tumor tissue and normal lung tissue were collected.

\section{Reverse transcription, quantitative real-time PCR and Western blot}

Reverse transcription, quantitative real-time PCR and Western blot were performed as described previously [33]. Oligonucleotide primers used for detection of human-SDPR, human-GAPDH (internal control), human-DACH1 and mouse-DACH1 were as follows: human-SDPR: $5^{\prime}$-CTCCGACGCAACCATTT-3'(sense); 5'-CTTTCTTGAGGCTATCCACTT-3' (antisense); human-GAPDH: 5'-AGAAGGTGGGGCTCATTTG-3' (sense); 5'-AGGGGCCATCCACAGTCTTC-3' (antisense); human-DACH1: 5'-GGAATGGATTGTGGC TGAAC- $3^{\prime} \quad$ (sense); $\quad 5^{\prime}$-GGTATTGGACTGGTACAT CAAG-3' (antisense); mouse-DACH1: 5'-AGTGGTGGT TCTTGGGATAAGG-3' (sense); 5'-TGAGAGGATGGC TAACTGGAA-3' (antisense) [34]. All the reactions were 
performed in triplicate for each sample. Cycle threshold (Ct) values of SDPR cDNA were normalized to GAPDH using the $-2 \Delta \Delta \mathrm{Ct}$ method. Western blot was performed according to standard protocols. These antibodies were used: SDPR (Proteintech, \#12339-1-AP; RRID:2183305), $\beta$-Actin (CST, \#8457; RRID:10950489). All the experiments were repeated three times.

\section{Clinical Specimens and Immunohistochemistry (IHC) staining}

Tissue microarray with clinical pathological data of lung cancer (HLugA180Su06) was purchased from Shanghai Outdo Biotech Biotechnology Co., Ltd., China. This lung cancer microarray (HLugA180Su06) contains 94 tumors and 86 paired adjacent normal tissues. All the tissues were collected from lung adenocarcinoma patients who underwent surgical resection from July 2004 to June 2009. The follow-up was from August 2014 and ranged from 5 to 10 years. IHC staining were performed as described in Additional file 1: S1 [31]. SDPR expression were detected with a rabbit polyclonal antibody against SDPR (Proteintech, \#12339-1-AP; RRID: 2183305).

\section{Screening of differentially expressed genes (DEGs) and identification of the abundance of tumor immune infiltration}

In this study, GSE18784, GSE49200, GSE72094 and GSE48414 were downloaded from GEO dataset, and datasets (PanCancer Atlas) contained lung adenocarcinoma expression profiles and paired normal tissues were downloaded from TCGA database through cBioPortal and sangerbox download tools. "EdgeR" $\mathrm{R}$ package in $\mathrm{R}$ version 3.6.2 (The R Foundation for Statistical Computing, Vienna, Austria; https://www.r-project.org/) was used to screen out the murine DEGs between normal and tumor tissues. "CIBERSORT.R" R package was used to explore the abundance of tumor immune infiltrations in KRAS-mutant lung adenocarcinomas, and TIMER 1.0 and 2.0 (Tumor Immune Estimation Resource, https:// cistrome.shinyapps.io/timer/) were used to identify the abundance of immune cells, such as B cells, $\mathrm{CD} 4^{+} \mathrm{T}$ cells, $\mathrm{CD}^{+} \mathrm{T}$ cells, Neutrophils, Macrophages and Dendritic cells at different SDPR copy number variation (CNV) patterns. The description of the above datasets and analysis processing method were described in Additional file 1: S2.

\section{Phylogenetic analysis of SDPR}

SDPR (NC_000002.12, gene ID 8436), also known as CAVIN2 is located in Chromosome 2. Homo sapiens amino acid sequences of $C A V$ and CAVIN family members were downloaded from Uniprot database. Subsequently MEGA-X (https:/www.megasoftware.net/) was used to conduct sequence alignment and infer phylogenetic trees (Additional file 1: S3). The phylogeny was inferred using the Neighbor-Joining method, and the tree is created and conducted using Interactive Tree Of Life (iTOL, https://itol.embl.de/).

\section{Bioinformatics mining of SDPR}

The information of chromosome location site and gene structure of SDPR gene were analyzed through GeneCards (https://www.genecards.org/). Protein sequences among $C A V I N$ and $C A V$ family members were downloaded from Uniprot database (https://www.unipr ot.org/). The sequence alignment was performed to analyze the identity between Homo sapiens, Mus musculus, Rattus norvegicus, Pan troglodytes, Macaca mulatta, Sus scrofa and Felis catus.

GEO dataset (GSE72094) and TCGA datasets (lung adenocarcinoma, PanCancer Atlas) and Gene Expression Profiling Interactive Analysis (GEPIA) database (https:// gepia.cancer-pku.cn/) were used to calculate the correlations between SDPR and Transcription factors (TFs), and evaluate overall survival (OS) of lung adenocarcinoma patients, under different SDPR expression levels and KRAS mutation status. TFs of SDPR were predicted using GeneCards (https://www.genecards.org/) and Promo (http://alggen.lsi.upc.es/cgi-bin/promo_v3/promo /promoinit.cgi?dirDB=TF_8.3), and microRNAs were predicted using miRanda (http://www.microrna.org/), miRDB (http://www.mirdb.org/) and TargetScan (http:// www.targetscan.org/vert_72/). The potential ceRNA network of SDPR in KRAS-mutant lung cancer was constructed using Cytoskype software. DAVID (https ://david.ncifcrf.gov/) and Gene Set Enrichment Analysis (GSEA,http://software.broadinstitute.org/gsea/regis ter.jsp) were used to perform the Gene Ontology (GO) enrichment analysis for biological process (BP), cellular component (CC), molecule function (MF) and pathways. "pheatmap" and "ggplot2" packages were used to visualize heatmaps and bubble charts.

\section{Statistical analysis}

All the data were analyzed by SPSS, version 20, IBM Corp., Armonk, USA. SDPR or DACH1 expression was presented as the mean \pm standard deviation (SD). Bars indicate SD. ${ }^{*} \mathrm{P}<0.05$; ${ }^{* *} \mathrm{P}<0.01$. Differences between the means were examined by student's test or one-way analysis of variance (ANOVA). Multiple comparisons among the groups were performed using LSD method. Nonparametric test was used to analyze the SDPR scores in lung and tumor tissues, and correlation analysis was assessed by Pearson or Spearman correlation method. KaplanMeier method and Cox proportional hazard regression model were used to evaluate the prognostic value of 
SDPR in lung adenocarcinoma. Differences with a value of $\mathrm{P}<0.05$ were considered statistically significant. All of the experiments were performed at least thrice.

\section{Results}

\section{The discovery of gene expression signature} in KRAS-oncogene-driven lung cancer

To uncover specific gene expression signature of KRASoncogene-driven lung cancer, we analyzed transcriptional expression profiles of normal lung tissues and $K R A S$-mutant lung tumor tissues based on GEO datasets
(GSE18784, GSE49200), respectively, and identified differentially expressed genes (DEGs) with statistical difference $(\mathrm{P}<0.05)$ between normal and tumor tissues. As shown in Fig. 1a, b, 25 upregulated DEGs and 45 downregulated DEGs were screened out based on GSE18784 dataset, using "EdgeR" R package. Using the same method, 155 upregulated DEGs and 120 downregulated DEGs were screened out based on GSE49200 dataset. The signatures between the two DEGs sets were different, indicating the heterogeneity of KRAS-driven tumors. Interestingly, SDPR was the only DEG that decreased

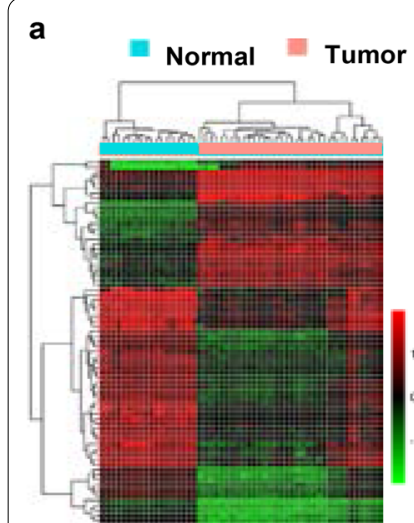

GSE18784

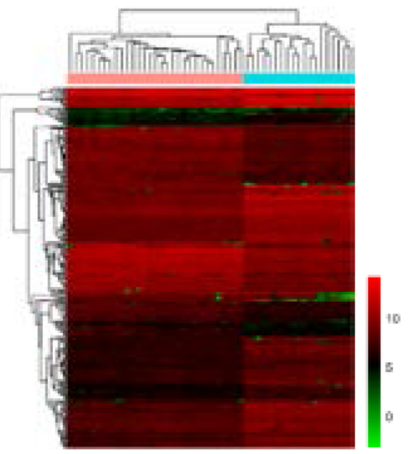

GSE49200

C

Chr 2

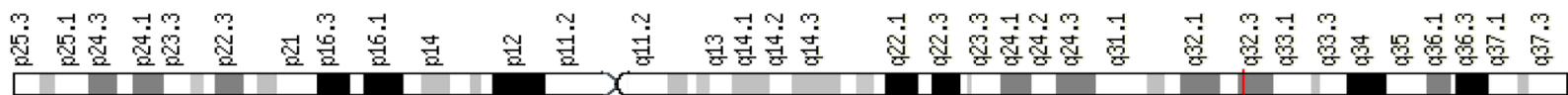

d

Homo sapiens
Mus musculus
Rattus norvegicus
Pan troglodytes
Macaca mulatta
Sus scrofa
Felis catus
(Species)
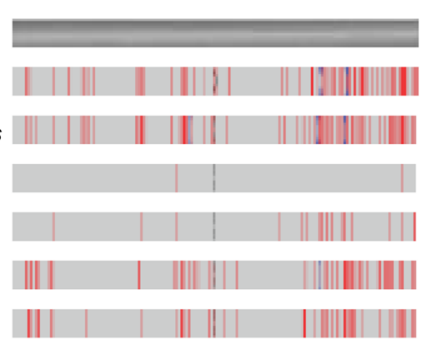

|||| || | || || |||| || ||||||||||| 425aa

418aa

417aa

425aa

425aa

424aa

425aa

(Length) b
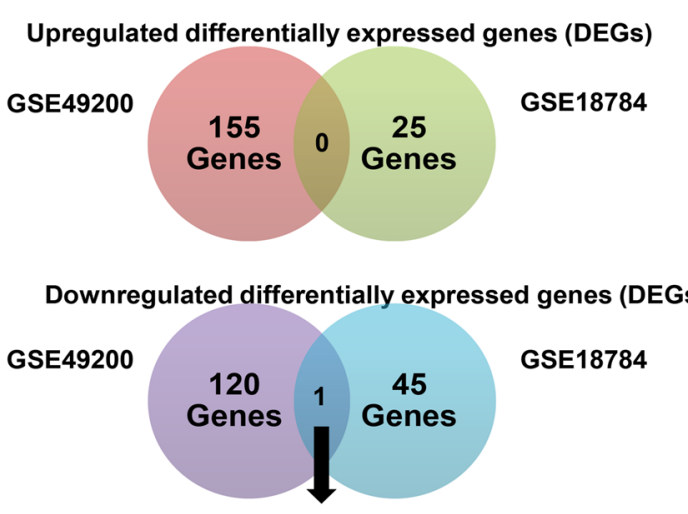

SDPR (CAVIN2; Gene ID: 8436)

Fig. 1 SDPR as a potential target for the treatment of KRAS-mutant lung cancer. a Transcriptional expression profiling in murine normal lung and tumor tissues with KRAS mutation based on GEO dataset (GSE18784, GSE49200). b The differentially expressed genes (DEGs) between murine normal and KRAS-mutant lung tumor tissues (normal vs tumor $=18$ vs 34 ; normal vs tumor $=19$ vs 31 , respectively). The gene structure and phylogenetic conservative analysis of SDPR. $\mathbf{c}$ Chromosome location of SDPR is marked in red. $\mathbf{d}$ Alignment analysis results of SDPR protein among Homo sapiens and other species is shown, and different sequences are marked in red. e Phylogenetic tree of Homo sapiens CAV and CAVIN family proteins including SDPR (highlighted in red frame). The tree was drawn to scale based on the above family proteins sequence (Uniprotdatabase), with branch lengths in the same units as those of the evolutionary distances used to infer the phylogenetic tree. The evolutionary distances were computed and visualized using MEGA software and iTOL website (https://itol.embl.de/) 
in KRAS-mutant tumor tissues based on GEO datasets (GSE18784, GSE49200), which suggested that the downregulation of SDPR might be a specific signature during the development of $K R A S$-mutant lung cancer.

\section{Structure and phylogenetic conservative analysis of SDPR} SDPR, also named CAVIN2, is a member of CAVIN family, which is located at chromosome 2, q32.3 (Fig. 1c). The structures of SDPR gene include 5'UTR exon, two exons, 3'UTR exon, and one intron. Protein sequences were compared to explore conservation of SDPR during molecule and species evolution, and the alignment results showed that Homo sapiens SDPR shared $82.82 \%$, 81.88\%, 99.53\%, 96\%, 87.29\% and $89.18 \%$ identity with Mus musculus, Rattus norvegicus, Pan troglodytes, Macaca mulatta, Sus scrofa, and Felis catus, respectively, which indicates that SDPR is highly conserved in mammals (Fig. 1d).

$C A V$ and CAVIN family members play important roles in the formation and stability of pulmonary alveoli [35]. Moreover, CAVIN members could regulate the expression of $C A V$ members. Thus, we analyzed the phylogenetic conservation of $C A V$ and $C A V I N$ family members. As shown in Fig. 1e, CAV and CAVIN family members are divided into two major clusters, and CAVIN2 shares a closer evolutionary relationship with CAVIN3, compared with CAVIN1 and CAVIN4.

\section{SDPR is downregulated in human lung adenocarcinoma, including KRAS-mutant group}

To identify the SDPR expression level in mouse and human lung tissues and tumors, we established KRASoncogene-driven lung cancer models [32] and detected SDPR expression using RT-qPCR. As shown in Fig. 2a, higher SDPR expression was detected in pulmonary than in bronchial tissue. Moreover, lower SDPR expression was observed in KRAS-mutant tumor tissues $(\mathrm{P}<0.05)$. We further confirmed SDPR expression in human tissues and found a similar result in KRAS-mutant tumors. As shown in Fig. 2b-e, SDPR expression significantly decreased in KRAS-mutant specimens as well as all lung tumors compared with normal tissue $(\mathrm{P}<0.05)$. In addition, low SDPR expression was detected in KRAS-mutant and KRAS-wild type NSCLC cell lines compared with immortalized normal lung cells, MRC-5 (Fig. 2f-g).

\section{Low expression of SDPR is associated with a poor prognosis in NSCLC patients}

As shown in Fig. 3a-c, low expression of SDPR was associated with shorter OS in NSCLC patients as well as in KRAS-mutant group, based on GEO dataset and lung cancer microarray (GSE72094, HLugA180Su06, P <0.05). Similar results were found in NSCLC patients using
GEPIA (Fig. 3d, $\mathrm{P}<0.05$ ). Meanwhile, univariate survival analysis indicated that low SDPR expression was associated with the shorter OS in NSCLC patients as well as in KRAS-mutant group (KRAS-mutant lung adenocarcinoma, $\mathrm{P}<0.05$, hazard ratio $[\mathrm{HR}]=0.7$; lung adenocarcinoma, $\mathrm{P}<0.05$, hazard ratio $[\mathrm{HR}]=0.7$; Table 1 ). Moreover, multivariate survival analysis showed that SDPR expression and stage were independent predictors of prognosis in lung adenocarcinoma patients as well as in KRAS-mutant group (Table 1). These data highlight the prognostic value of SDPR in human lung adenocarcinoma, especially in $K R A S$-mutant subgroup.

\section{Construction of competing endogenous RNA (ceRNA) network of SDPR in KRAS-mutant lung adenocarcinoma pathway}

To identify the upstream regulatory structure of SDPR in KRAS-mutant lung cancer, DEGs based on GSE72094 and three public predicted websites (TargetScan, miRDB and miranda) were used (Fig. 4a). Briefly, 139 expression profiles of KRAS-mutant patients with complete clinical information were collected (GSE72094), and DEGs sets between low and high SDPR group were screened out using "EdgeR" R package. Three public websites, TargetScan, miRDB and miranda, were used to predict potential combinations between SDPR and transcription factors. As shown in Fig. 4a, two transcription factors (DACH1, WT-1) were identified based on DEGs and TargetScan websites. Moreover, SDPR correlated positively with DACH1 $\left(R^{2}=0.509, P<0.01\right.$; Fig. $\left.4 b\right)$ and negatively with WT-1 $\left(R^{2}=-0.218, P<0.05\right.$; Fig. $\left.4 c\right)$. We detected the expression of DACH1 in NSCLC cell lines, MRC5 cells and the KRAS-oncogene-driven lung cancer mice. The DACH1 expression in bronchial tissue was lower than that in normal lung tissue based on KRAS oncogenic mice models. Meanwhile, DACH1 expression was lower in tumor tissue than in normal lung tissue. Moreover, The DACH1 expression in NSCLC cells was lower than that in MRC5 cells (Additional file 1: Figure S1).

Similar to the above screening method of transcription factors, a set of miRNAs was predicted, and five miRNAs (hsa-miR-1, hsa-miR-204, hsa-miR-144, hsa-miR-105 and hsa-miR-363) were ultimately screened out, which were observed in the above 4 miRNA sets (Fig. 4d). All of them were downregulated in KRAS-mutant lung adenocarcinoma compared with normal lung tissues (Fig. 4e). Interestingly, we found some potential complementary sequences between hsa-miR-1 and DACH-1 (Fig. 4f), indicating that the above miRNAs and TFs may form a complex network to regulate SDPR expression. Thus, we screened a series of miRNAs with potential combination sequence with SDPR-related TFs, and constructed a 


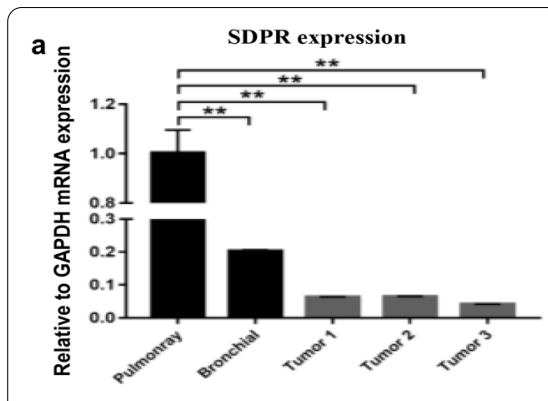

b

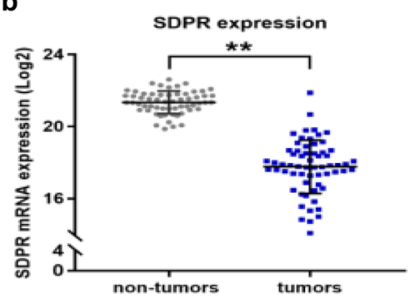

e

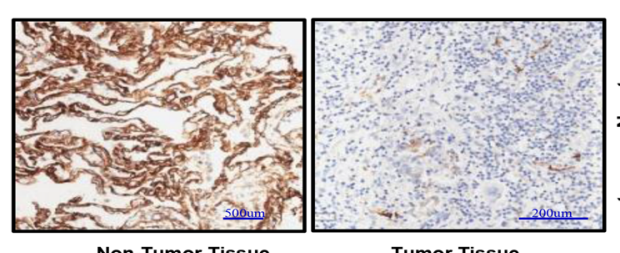

Non-Tumor Tissue

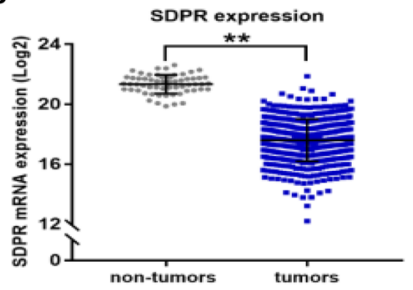

SDPR expression

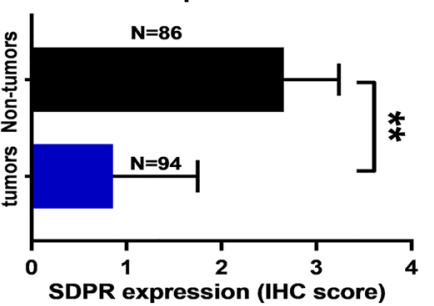

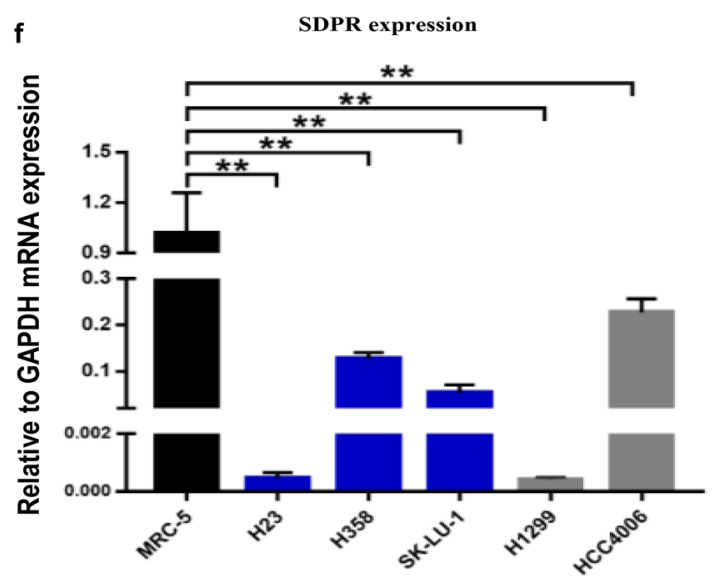

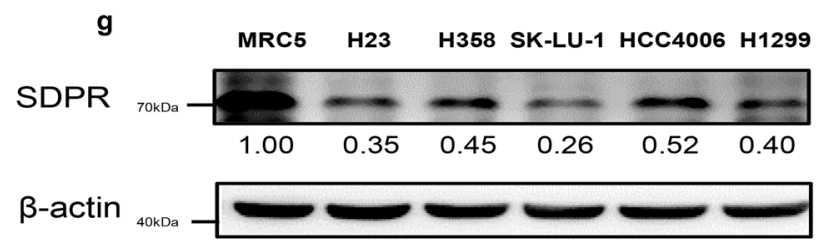

Fig. 2 SDPR expression level is downregulated in lung cancers as well as in KRAS-mutant group. a SDPR expression in murine normal lung tissue and KRAS-mutant tumors measured by RT-qPCR. b SDPR expression level in tumor and normal tissue in the KRAS-mutant subgroup based on TCGA database. c SDPR expression level in lung tumor and normal tissue based on TCGA database. $\mathbf{d}$ SDPR expression level in lung tumor and normal tissue based on GEPIA. e SDPR expression in 180 lung tumor and normal tissue measured by immunohistochemistry (IHC). f, $\mathbf{g}$ SDPR expression in human embryonal lung cells, KRAS-mutant and KRAS wild-type lung cancer cells measured by RT-qPCR and western blot, and the results of western blot were quantified using Image J quantitative analysis software. Bars indicate $S D$. ${ }^{*} \mathrm{P}<0.05 ;{ }^{* *} \mathrm{P}<0.01$. All of the experiments were repeated three times

competing endogenous RNA (ceRNA) network of SDPR in KRAS-mutant lung adenocarcinoma (Fig. 4g).

\section{Biological enrichment analysis of SDPR downstream pathway}

To explore the downstream pathway of SDPR, DEGs based on GSE72094 were explored to identify biological differences between tissues with low and high SDPR expression in KRAS-mutant lung cancer. Gene ontology analysis was performed using DAVID online software to unfold the biological function of biological process, cellular component and molecule function among the above DEGs. As shown in Fig. 5a-c, biological processes were mainly associated with cell mitosis and cell cycle, and the differences of cellular components were mainly located in the extracellular space, exosomes, and matrix. In addition, there were a series of members related to redox balance and energy transfer, indicating the close interaction between SDPR expression and metabolism. Moreover, GSEA analysis results showed that G2 pathway and TGF-beta pathway were most likely associated with the above DEGs (Fig. 5d, e). 

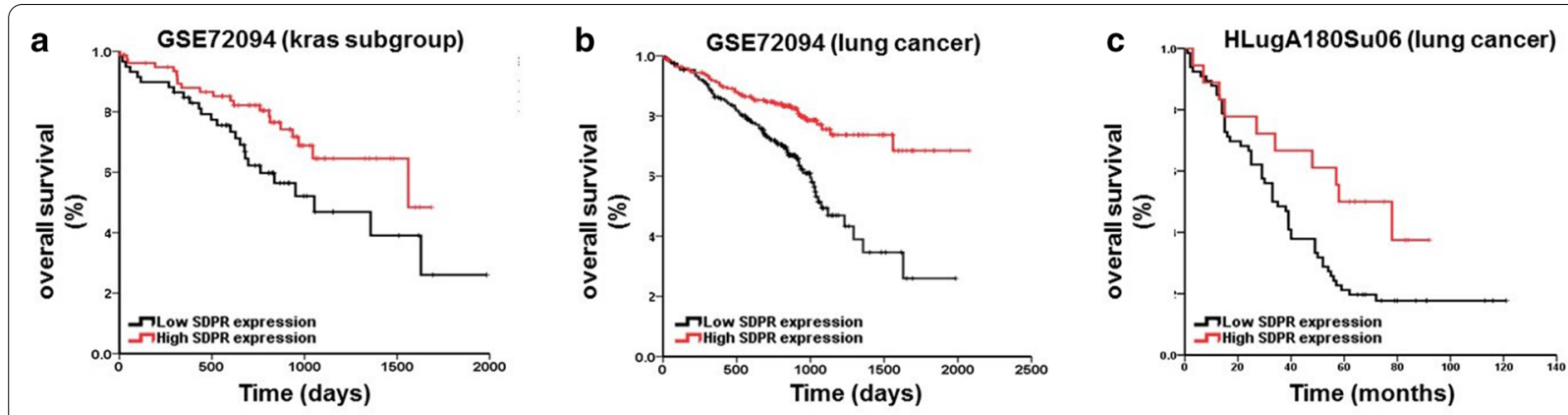

d
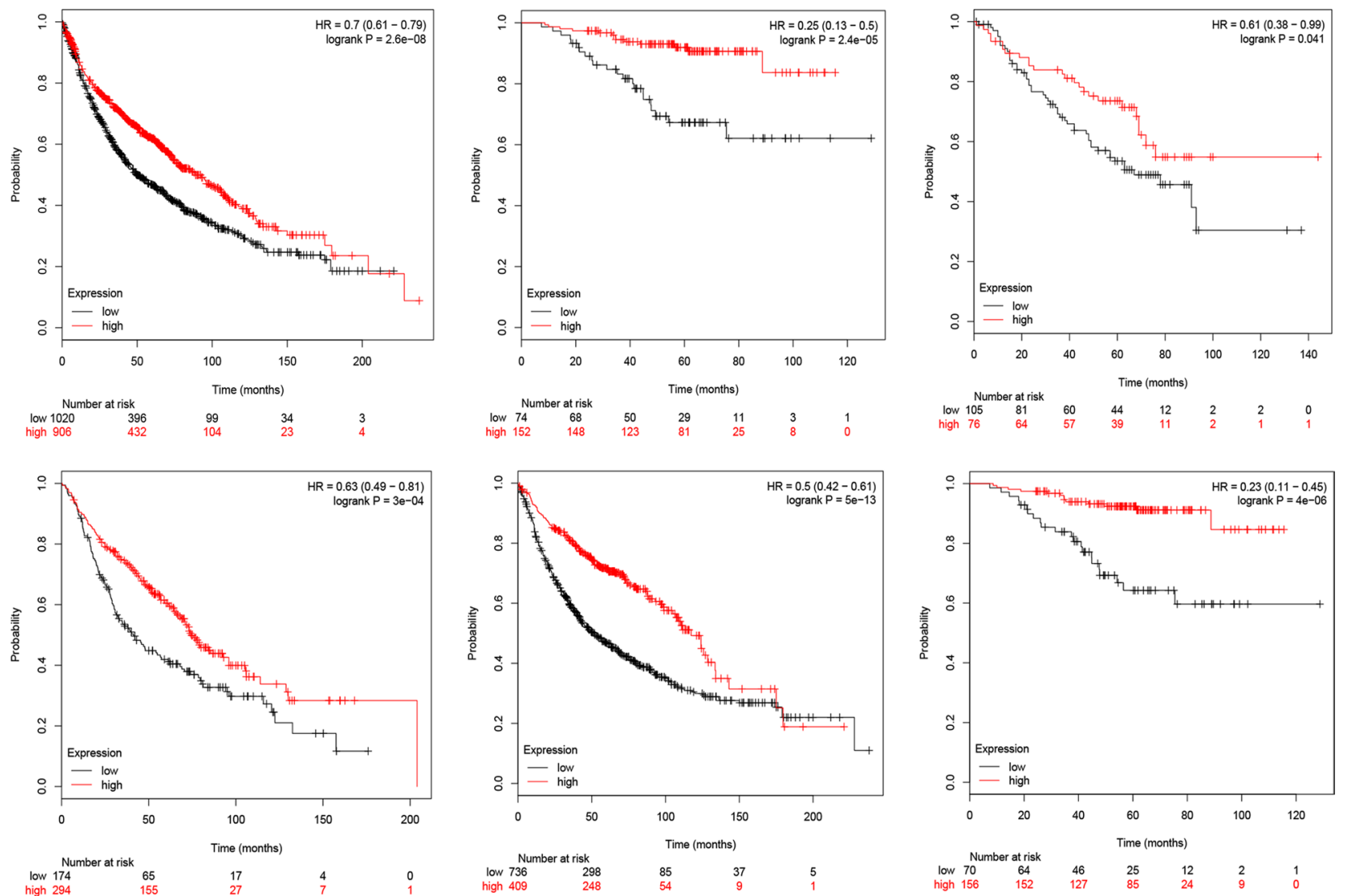

Fig. 3 Prognostic values of SDPR in lung adenocarcinoma, as well as in KRAS-mutant subtype. a, $\mathbf{b}$ Impact of SDPR expression on the overall survival in KRAS-mutant and all of the lung adenocarcinoma patients based on GEO dataset (GSE72094). c Impact of SDPR expression on the overall survival in lung adenocarcinoma patients based on lung cancer microarray (HLugA180Su06). $\mathbf{d}$ Impact of SDPR expression on the overall survival in lung adenocarcinoma patients based on the cohorts downloaded from GEPIA

\section{Correlation between SDPR, immune negative regulatory} molecules and immune infiltration models

Recently, SDPR was reported to play an important role in cancer progression and metastasis via epithelial mesenchymal transition (EMT) in gastric and breast cancers $[27,36]$. However, the function of SDPR in lung cancer, especially in $K R A S$-mutant group, remains unclear. Since different SDPR expression levels are accompanied with changes in extracellular components (Fig. 5c), we hypothesized that SDPR expression may be closely related with tumor environment. Thus, we explored the correlation between SDPR, immune checkpoint molecules and immune infiltration models.

As shown in Fig. 6a, SDPR expression level correlated negatively with PD-L1(CD274), GITR(TNFRSF18), 4-1BBR(TNFRSF9) and TDO2 $\left(\mathrm{R}^{2}=-0.247,-0.327\right.$, $-0.183,-0.233$, respectively; $\mathrm{P}<0.05)$. Since the role of SDPR in immune infiltration is unclear, we analyzed 
Table 1 Impact of SDPR expression and clinic pathologic characteristics in lung adenocarcinoma

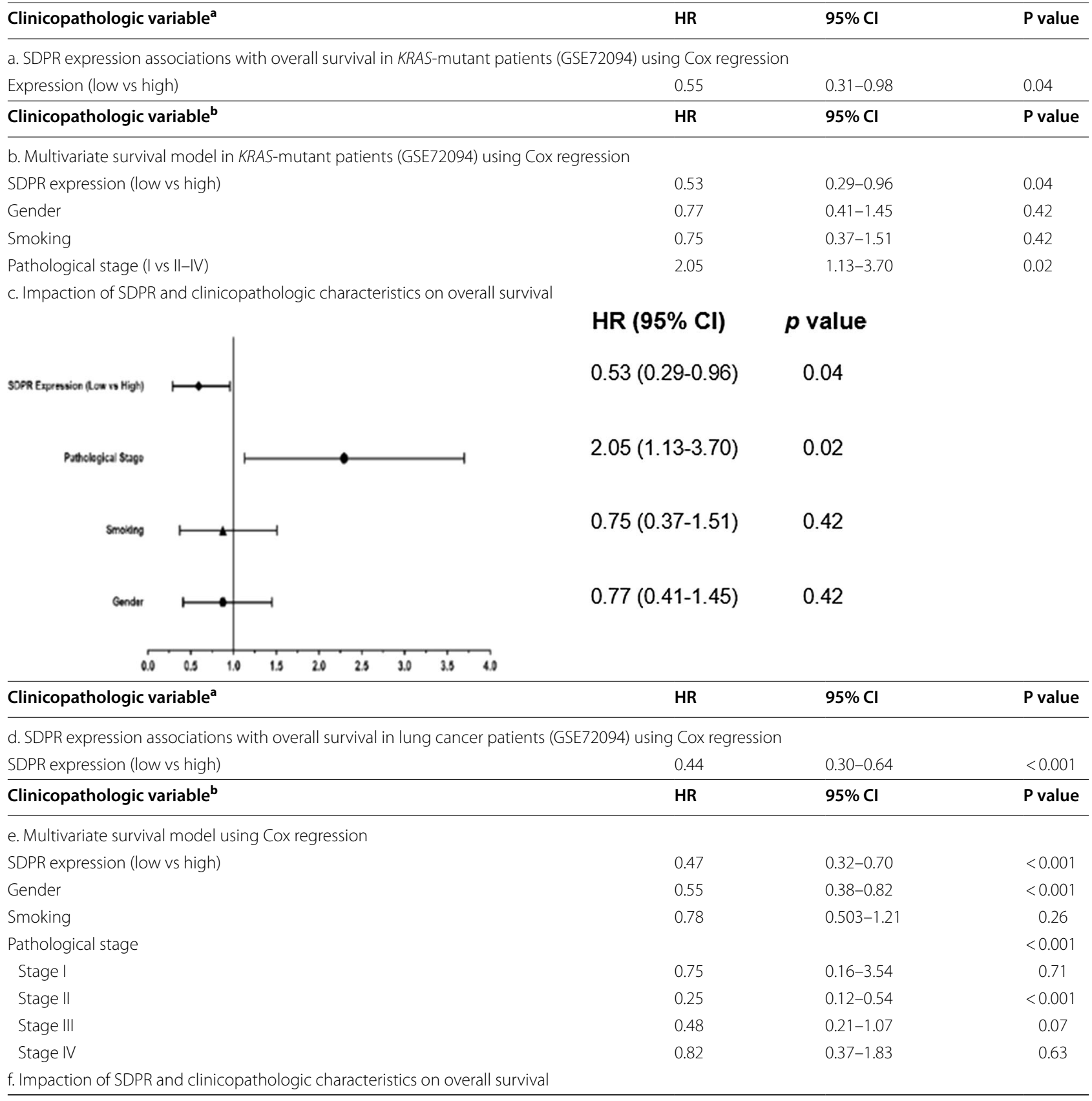


Table 1 (continued)

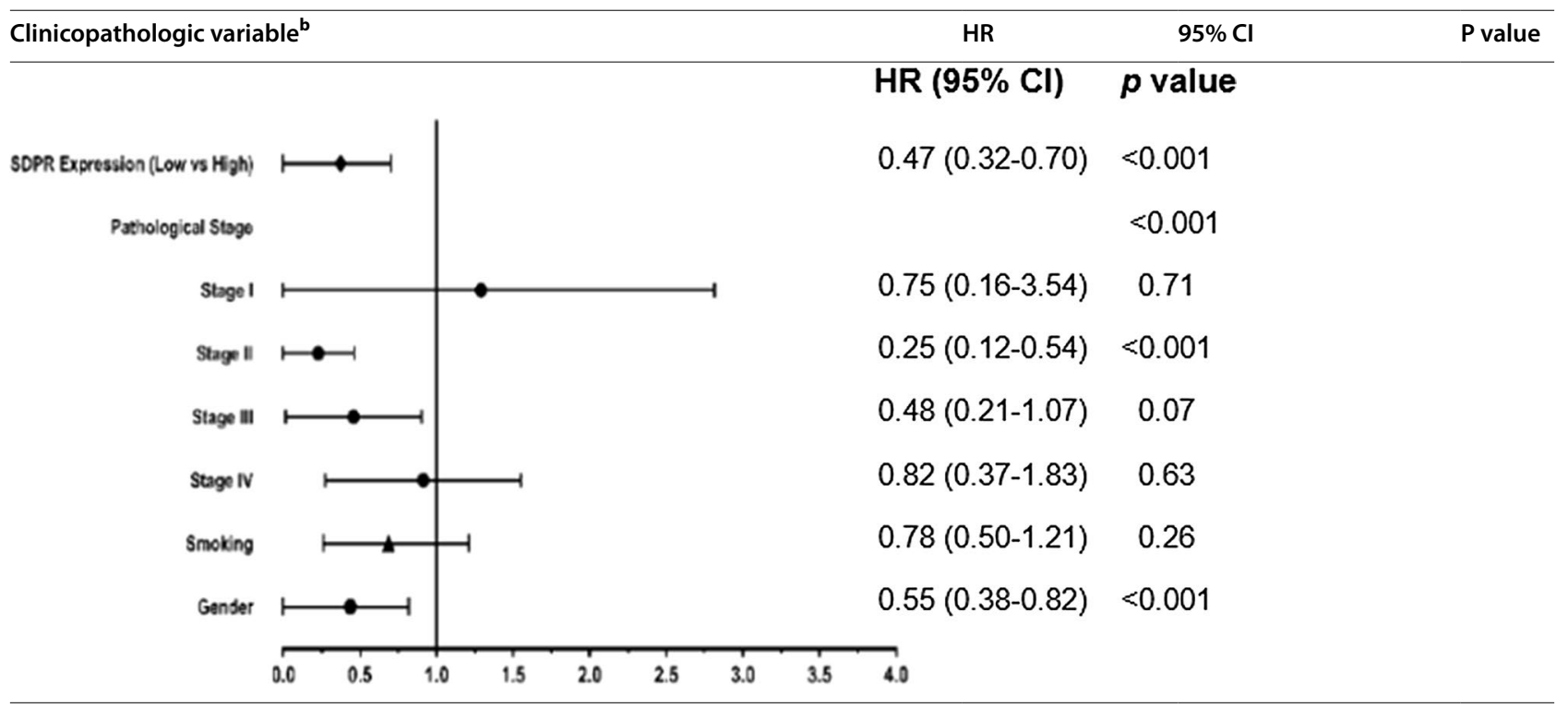

the abundance of immune cells in lung cancers at different SDPR expression levels and copy number variation (CNVs) patterns. In KRAS-mutant subgroups, cancer tissue with lower expression of SDPR was accompanied with less infiltration of $\gamma \mathrm{T}$ cells and resting mast cells but higher abundance of plasma cells, $\mathrm{CD}^{+}$memory activated $\mathrm{T}$ cells and M1 macrophages (Fig. 6b). Meanwhile, SDPR expression in lung adenocarcinoma correlated positively with infiltration of memory B cells, endothelial cells, M1 and M2 macrophages, myeloid dendritic cells, neutrophils, memory resting $\mathrm{CD} 4^{+} \mathrm{T}$ cells, $\mathrm{CD}^{+} \mathrm{T}$ cells, but correlated negatively with M0 macrophages, plasma $\mathrm{B}$ cells, and $\mathrm{CD}^{+}$memory activated $\mathrm{T}$ cells based on TIMER 2.0 website (Table 2). In addition, lung adenocarcinoma with SDPR arm-level deletion showed less infiltration of $\mathrm{CD} 4^{+} \mathrm{T}$ cells, macrophages and neutrophils in TME (Fig. 6c).

These results illustrated close relationship between SDPR, PD-L1(CD274), GITR(TNFRSF18), 4-1BBR(TNFRSF9), TDO2, and abundance of immune cells in human lung adenocarcinoma, especially in KRASmutant subgroups.

\section{Discussion}

Over 8 different variants of KRAS mutation have been identified at codons 12, 13 and 61 in NSCLC [37]. Several studies explored the therapeutic vulnerability and prognostic differences between the KRAS mutation subtypes [38-40]. However, KRAS mutant status may not be recommended to select NSCLC patients for specific treatment such as adjuvant chemotherapy. Meanwhile, there were no significant differences in the phosphorylation level of MEK/ERK kinase among the above variants, despite phosphorylation of AKT and activation of RAL seem to differ between KRAS-G12C and KRAS-G12V cells $[4,38]$. In summary, no specific $K R A S$ variants were validated as ideal prognostic factors of survival or vulnerability indicators for treatment of KRAS-mutant tumors. In our study, we found that SDPR expression was not only decreased in KRAS-mutant NSCLC cells, and KRAS-driven murine tumor from GEMMs, but also downregulated in human NSCLC specimens based on GEO datasets, TCGA datasets, and lung adenocarcinoma tissue array (Fig. 2a-g). Moreover, SDPR expression was suggested to be an independent prognostic factor in lung cancer (Fig. 3a-d, Table 1). Our research provides a potential target for the prognosis and treatment of NSCLC independent of KRAS variants. More biological experiments and clinical trials are needed to validate and complement our conclusions.

Co-occurring genetic events were frequently observed in KRAS-mutant lung tumors, unlike other oncogenedriven lung cancers $[40,41]$. STK11 co-mutations (KL), TP53 co-mutations (KP), and CDKN2A/B inactivation plus low thyroid transcription factor-1 (TTF-1) expression $(\mathrm{KC})$ were considered as classical models among $K R A S$-mutant tumors, and may induce different biological behaviors and characteristics of tumors [40, 42]. Cooccurring STK11 or KEAP1 mutations were associated with worse OS in KRAS-mutant NSCLCs [9, 40]. Moreover, the lowest levels of PD-L1 and deficient inflammatory immune cells infiltration were found in the KL group. In contrast, the KP group with the highest PD-L1 expression was infiltrated with active inflammation (mainly 
a

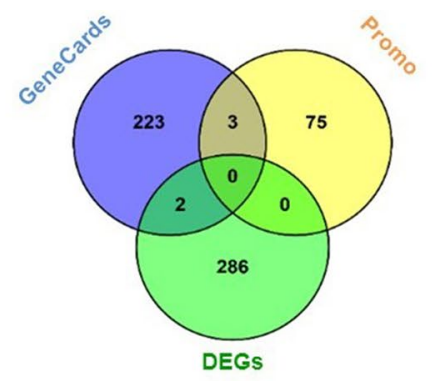

d

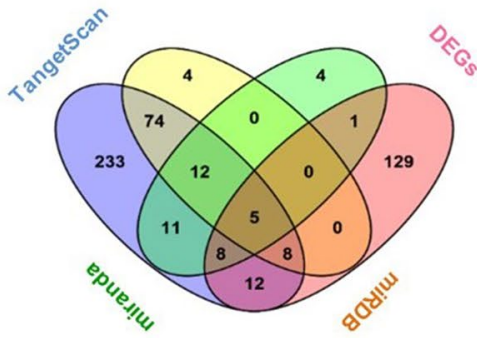

$\mathbf{f}$

hsa-miR-1: 3' uaugUAUGA--AGAAAUGUAAGGu 5' SDPR(1036site): 5' auUUAAACUAACCCUUACAUUCCa 3 '

hsa-miR-1: 3' uaUGUAUGA -- AGAAAUGUAAGGu 5' SDPR(1136site): 5' gUACAUAAUGGGAUUUCCAUUCC 3 '

hsa-miR-1 : 3' uauguaUGAAGAAAUGUAAGGu 5' DACH1(716site): 5' aguguaACAUGUUUUCAUUCCa 3'

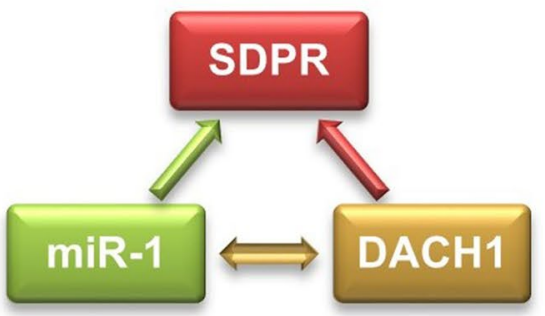

b

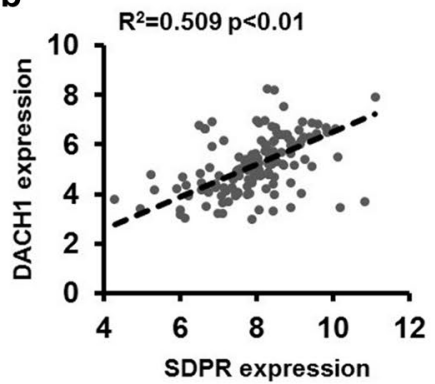

C

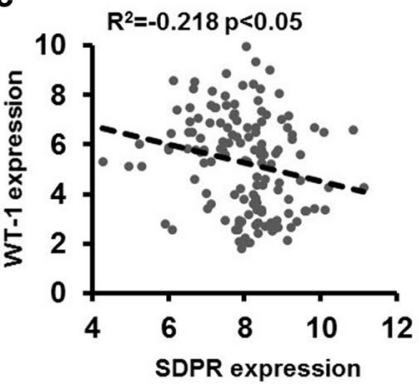

e

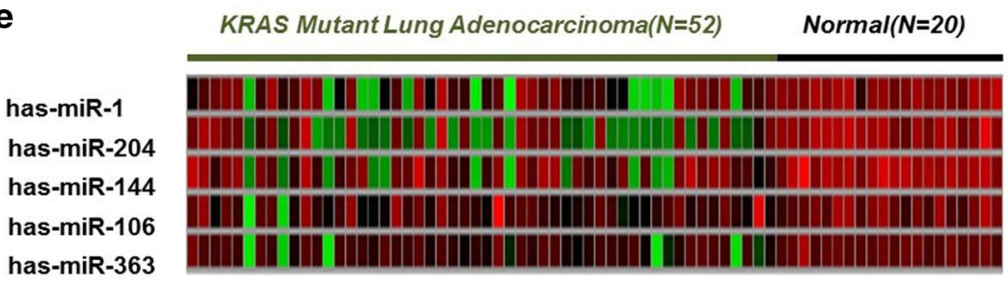

g

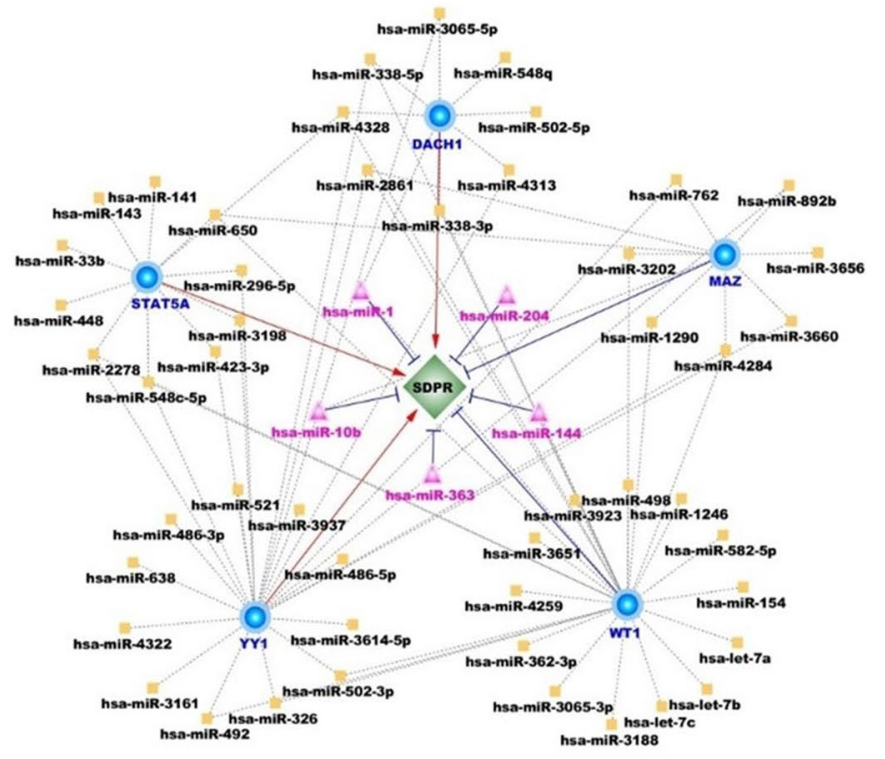

Fig. 4 Construction of ceRNA network of SDPR in KRAS-mutant lung adenocarcinoma. a Prediction of upstream transcription factors (TFs) of SDPR in KRAS-mutant lung adenocarcinoma. KRAS-mutant specific TFs were screened out based on GEO dataset (GSE72094), and intersected with SDPR related TFs predicted by online database (Promo or Genecards). b The correlations between SDPR and two predicted TFs, DACH1 and WT-1 based on TCGA dataset (Atlas). c, d Prediction of upstream miRNAs of SDPR in KRAS-mutant lung adenocarcinoma. KRAS-mutant specific miRNAs were screened out based on TCGA dataset (GSE48414), then intersected with SDPR combined miRNAs predicted by miRDB, miranda and targetScan. Five miRNAs finally were screened out and the expression levels in normal tissue and KRAS-mutant lung carcinoma were shown with heat map. $\mathbf{f}$ Complementary sequences between hsa-miR-1, DACH1 and SDPR. $\mathbf{g}$ Construction of ceRNA network to visualize the regulation models of SDPR, TFs and miRNA in KRAS-mutant lung carcinoma 


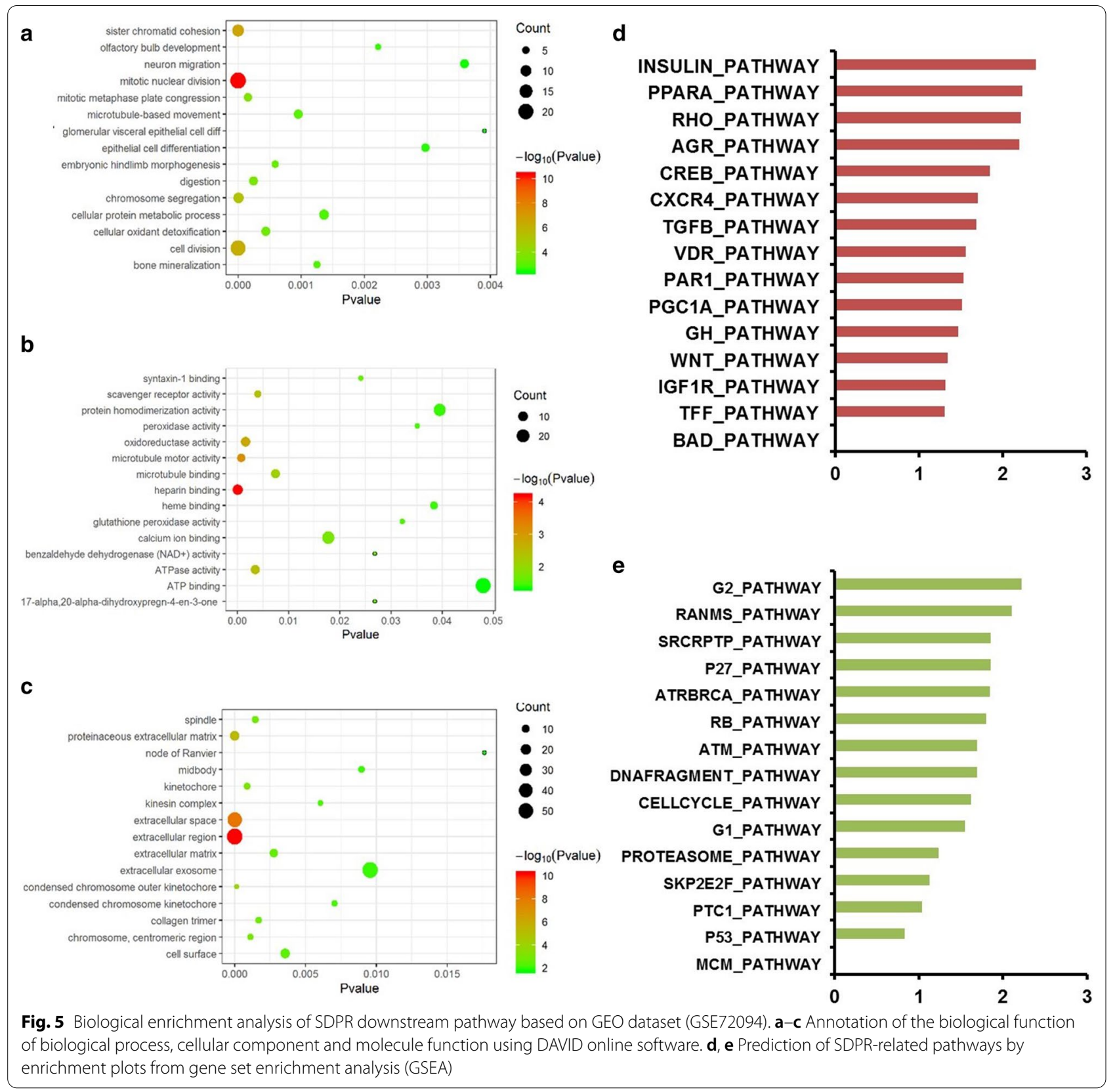

$\mathrm{T}$ cell inflammation) [24]. Our research found negative correlations between SDPR, PD-L1, and immune cells in $K R A S$-mutant lung cancers (Fig. 6a-c). More studies should explore the influence of co-occurring genetic events on SDPR expression and malignant biological behaviors of tumors.

Previous research explored the prognostic and diagnostic significance of SDPR in gastric cancer [27], hepatocellular carcinoma [28], and papillary thyroid cancer (PTC) [29]. Our research originally found downregulation of SDPR in lung cancers as well as in KRAS-mutant subgroup (Fig. 2a-g), and innovatively explored the immune checkpoint molecules and abundance of immune infiltrations at different SDPR expression and CNVs models (Fig. 6a-c, Table 2). Those results provide a novel theory for the immune regulatory functions of SDPR in tumorigenesis, progression and metastasis.

In terms of the regulation and function of SDPR in lung cancer, the reason leads to the depression of SDPR is unclear. It was reported that MiR-577 regulates TGF- $\beta$ in gastric cancer through a SDPR-modulated positive-feedback loop [27]. Moreover, overexpression 

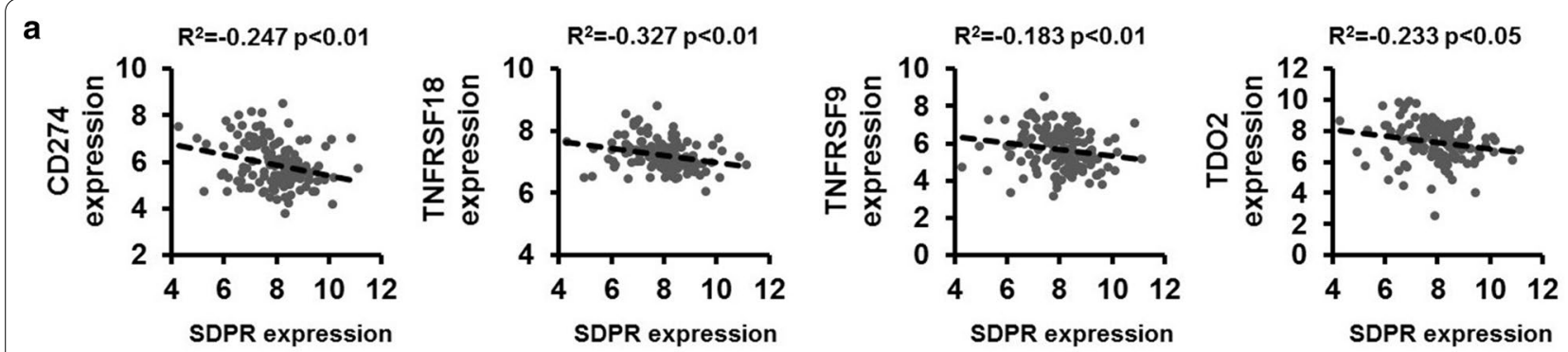

b

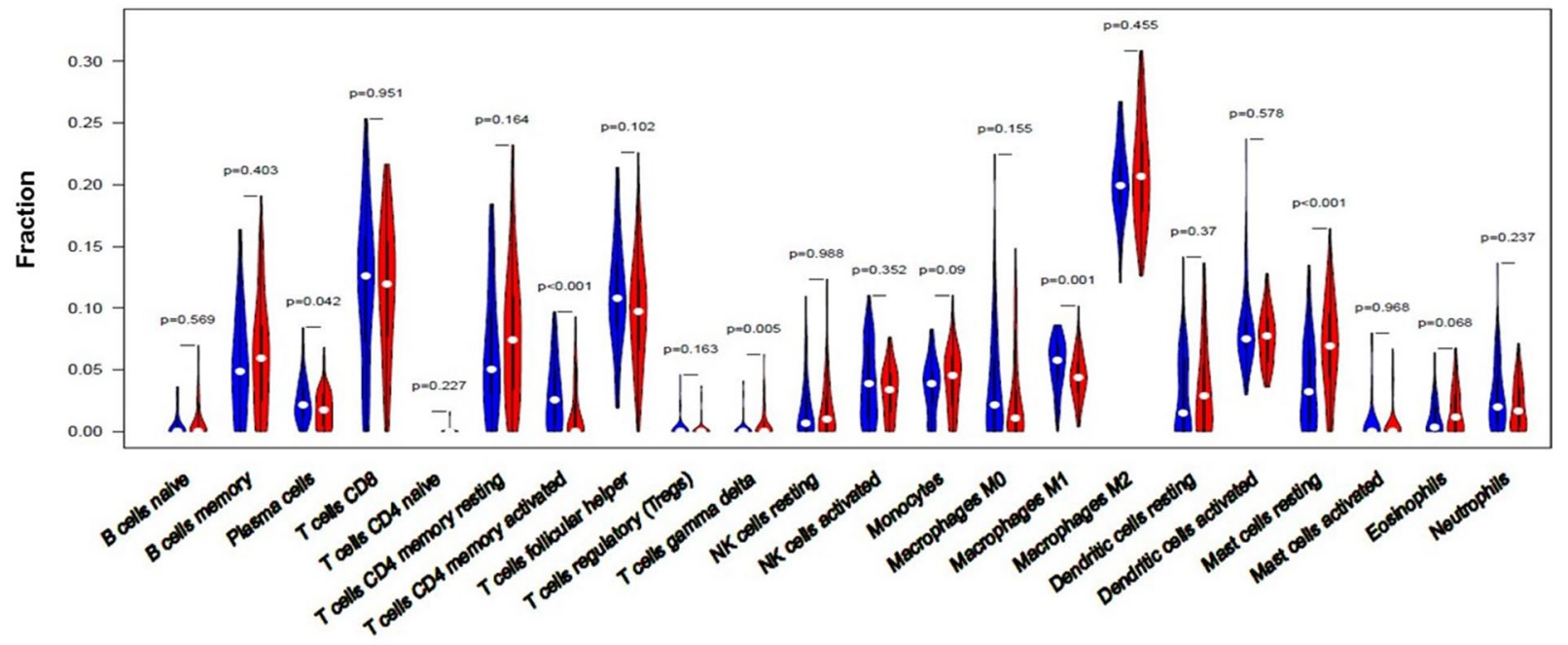

C

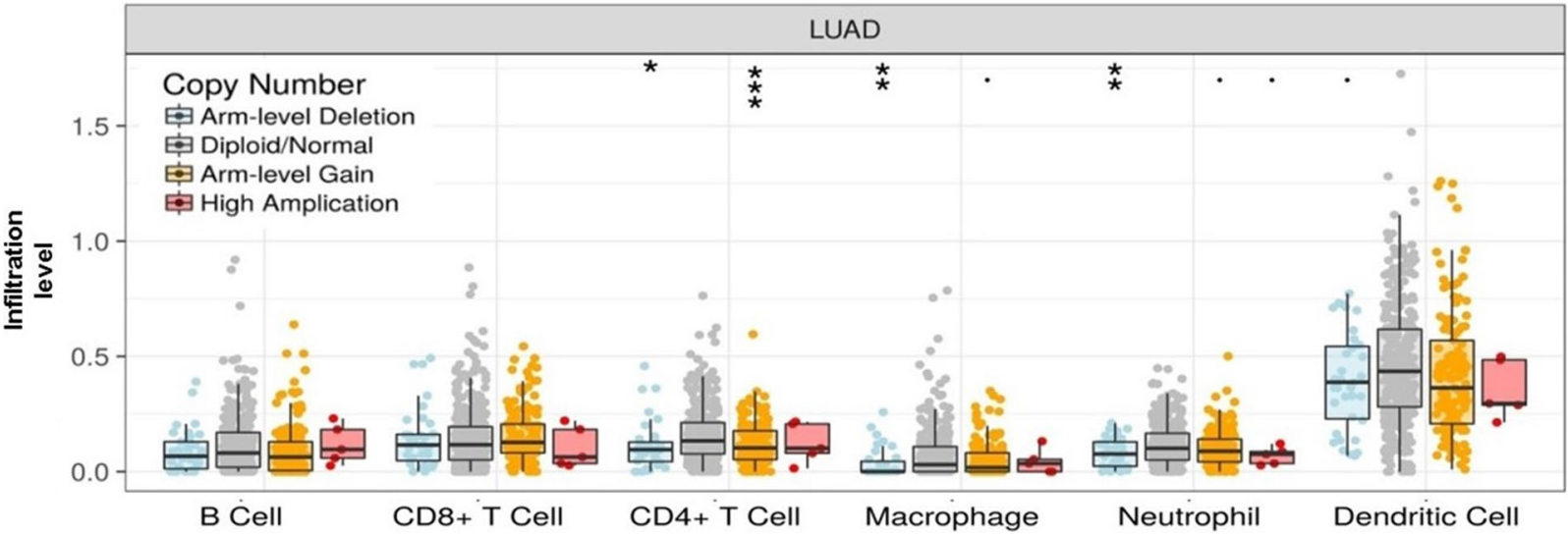

Fig. 6 Correlation between SDPR, immune checkpoint molecules and abundance of tumor immune infiltrations. a Correlations between SDPR expression and immune checkpoint molecules based on GEO dataset (GSE72094). b Abundance of immune infiltrates in KRAS-mutant lung cancer with different somatic CNV patterns of SDPR based on GEO dataset (GSE72094). Low SDPR expression group marked in blue, and high SDPR expression group marked in red. c Correlations between SDPR mRNA expression and abundance of immune infiltrates in lung adenocarcinoma using TIMER (comprehensive resource for the clinical relevance of tumor-immune infiltrations) system. $\mathbf{d}$ Abundance of immune infiltrates in lung adenocarcinoma with different somatic CNV patterns of SDPR using TIMER system

of SDPR inhibited the activity of ERK and NF- $\kappa$ B pathways in breast cancer [36]. In our study, a series of pathways, including the TGF- $\beta$ pathway, were enriched between SDPR-low and SDPR-high specimens in KRAS mutant lung cancers (Fig. 5d). In addition, results of GO analysis indicated different distribution of extracellular components depending on SDPR expression (Fig. 5c). Our study screened out a series of TFs and miRNAs as promising candidates for the upstream targets of SDPR in KRAS-mutant cancers, and we constructed a ceRNA 
Table 2 Correlation between SDPR expression and immune infiltration in lung adenocarcinoma

\begin{tabular}{|c|c|c|c|}
\hline Infiltrates & rho & $\mathbf{p}$ & adj.p \\
\hline B cell memory_CIBERSORT & $\underline{0.14}$ & 0.00 & 0.00 \\
\hline B cell memory_CIBERSORT-ABS & $\underline{0.18}$ & 0.00 & 0.00 \\
\hline B cell plasma_XCELL & -0.21 & 0.00 & 0.00 \\
\hline B cell_EPIC & $\underline{0.10}$ & 0.03 & 0.07 \\
\hline B cell_MCPCOUNTER & $\underline{0.15}$ & 0.00 & 0.00 \\
\hline B cell_QUANTISEQ & $\underline{0.12}$ & 0.01 & 0.02 \\
\hline Cancer associated fibroblast_EPIC & -0.09 & 0.04 & 0.10 \\
\hline Cancer associated fibroblast_XCELL & $\underline{0.36}$ & 0.00 & 0.00 \\
\hline Common lymphoid progenitor_XCELL & -0.21 & 0.00 & 0.00 \\
\hline Common myeloid progenitor_XCELL & $\underline{0.17}$ & 0.00 & 0.00 \\
\hline Endothelial cell_EPIC & $\underline{0.51}$ & 0.00 & 0.00 \\
\hline Endothelial cell_MCPCOUNTER & $\underline{0.61}$ & 0.00 & 0.00 \\
\hline Endothelial cell_XCELL & $\underline{0.47}$ & 0.00 & 0.00 \\
\hline Eosinophil_XCELL & $\underline{0.15}$ & 0.00 & 0.00 \\
\hline Granulocyte-monocyte progenitor_XCELL & $\underline{0.39}$ & 0.00 & 0.00 \\
\hline Hematopoietic stem cell_XCELL & $\underline{0.62}$ & 0.00 & 0.00 \\
\hline Macrophage MO_CIBERSORT & -0.33 & 0.00 & 0.00 \\
\hline Macrophage MO_CIBERSORT-ABS & -0.22 & 0.00 & 0.00 \\
\hline Macrophage M1_CIBERSORT & -0.12 & 0.01 & 0.03 \\
\hline Macrophage M1_QUANTISEQ & $\underline{0.24}$ & 0.00 & 0.00 \\
\hline Macrophage M2_CIBERSORT & $\underline{0.22}$ & 0.00 & 0.00 \\
\hline Macrophage M2_CIBERSORT-ABS & $\underline{0.38}$ & 0.00 & 0.00 \\
\hline Macrophage M2_QUANTISEQ & $\underline{0.40}$ & 0.00 & 0.00 \\
\hline Macrophage M2_XCELL & $\underline{0.29}$ & 0.00 & 0.00 \\
\hline Macrophage_EPIC & $\underline{0.14}$ & 0.00 & 0.01 \\
\hline Macrophage_TIMER & $\underline{0.19}$ & 0.00 & 0.00 \\
\hline Macrophage_XCELL & $\underline{0.10}$ & 0.03 & 0.07 \\
\hline Mast cell activated_CIBERSORT & $\underline{0.38}$ & 0.00 & 0.00 \\
\hline Mast cell activated_CIBERSORT-ABS & $\underline{0.42}$ & 0.00 & 0.00 \\
\hline Mast cell resting_CIBERSORT & -0.23 & 0.00 & 0.00 \\
\hline Mast cell resting_CIBERSORT-ABS & -0.20 & 0.00 & 0.00 \\
\hline Mast cell_XCELL & $\underline{0.32}$ & 0.00 & 0.00 \\
\hline MDSC_TIDE & -0.50 & 0.00 & 0.00 \\
\hline Monocyte_CIBERSORT & $\underline{0.34}$ & 0.00 & 0.00 \\
\hline Monocyte_CIBERSORT-ABS & $\underline{0.40}$ & 0.00 & 0.00 \\
\hline Monocyte_QUANTISEQ & -0.22 & 0.00 & 0.00 \\
\hline Monocyte_XCELL & $\underline{0.18}$ & 0.00 & 0.00 \\
\hline Myeloid dendritic cell activated_CIBERSORT & $\underline{0.13}$ & 0.00 & 0.01 \\
\hline Myeloid dendritic cell activated_CIBERSORT-ABS & $\underline{0.17}$ & 0.00 & 0.00 \\
\hline Myeloid dendritic cell activated_XCELL & $\underline{0.15}$ & 0.00 & 0.00 \\
\hline Myeloid dendritic cell resting_CIBERSORT & $\underline{0.13}$ & 0.00 & 0.01 \\
\hline Myeloid dendritic cell resting_CIBERSORT-ABS & $\underline{0.17}$ & 0.00 & 0.00 \\
\hline Myeloid dendritic cell_MCPCOUNTER & $\underline{0.29}$ & 0.00 & 0.00 \\
\hline Myeloid dendritic cell_QUANTISEQ & -0.19 & 0.00 & 0.00 \\
\hline Myeloid dendritic cell_TIMER & $\underline{0.17}$ & 0.00 & 0.00 \\
\hline Myeloid dendritic cell_XCELL & $\underline{0.29}$ & 0.00 & 0.00 \\
\hline Neutrophil_MCPCOUNTER & $\underline{0.33}$ & 0.00 & 0.00 \\
\hline Neutrophil_QUANTISEQ & $\underline{0.18}$ & 0.00 & 0.00 \\
\hline Neutrophil_TIMER & $\underline{0.11}$ & 0.01 & 0.03 \\
\hline
\end{tabular}

Table 2 (continued)

\begin{tabular}{lrll}
\hline Infiltrates & rho & p & adj.p \\
\hline NK cell activated_CIBERSORT-ABS & $\underline{0.09}$ & 0.04 & 0.10 \\
NK cell_EPIC & -0.10 & 0.03 & 0.07 \\
Plasmacytoid dendritic cell_XCELL & -0.15 & 0.00 & 0.00 \\
T cell CD4+(non-regulatory)_XCELL_ & $\underline{0.09}$ & 0.04 & 0.08 \\
T cell CD4+ effector memory_XCELL & $\underline{0.12}$ & 0.01 & 0.02 \\
T cell CD4+ memory activated_CIBERSORT & -0.24 & 0.00 & 0.00 \\
T cell CD4+ memory activated_CIBERSORT-ABS & -0.23 & 0.00 & 0.00 \\
T cell CD4+memory resting_CIBERSORT & $\underline{0.28}$ & 0.00 & 0.00 \\
T cell CD4+memory resting_CIBERSORT-ABS & $\underline{0.37}$ & 0.00 & 0.00 \\
T cell CD4+Th1_XCELL & -0.38 & 0.00 & 0.00 \\
T cell CD4+Th2_XCELL & -0.38 & 0.00 & 0.00 \\
T cell CD4+_EPIC & $\underline{0.30}$ & 0.00 & 0.00 \\
T cell CD8 + naive_XCELL & -0.21 & 0.00 & 0.00 \\
T cell CD8 +_CIBERSORT-ABS & $\underline{0.16}$ & 0.00 & 0.00 \\
T cell CD8 +_EPIC & $\underline{0.24}$ & 0.00 & 0.00 \\
T cell CD8 +_TIMER & $\underline{0.17}$ & 0.00 & 0.00 \\
T cell follicular helper_CIBERSORT & -0.11 & 0.01 & 0.03 \\
T cell regulatory (Tregs)_CIBERSORT & -0.17 & 0.00 & 0.00 \\
T cell regulatory (Tregs)_QUANTISEQ & $\underline{0.34}$ & 0.00 & 0.00 \\
\hline Significant correlation between immune cell subgroups and SDPR expression \\
were shown in Table 2 based on TIMER, CIBERSORT, quanTIseq, xCell, MCP- \\
counter and EPIC algorithms. Positive correlation was marked in underline, while \\
negative correlation was marked in italics. P<0.01 were marked as 0.00 &
\end{tabular}

regulation network of SDPR in KRAS mutant lung cancers, which provided useful information for the molecule regulatory network of SDPR in KRAS-mutant lung cancers.

\section{Conclusions}

In our study, a decrease of SDPR was found in lung cancers as well as in KRAS-mutant subgroup, and which may be a promising prognostic marker for the survival of patients with lung cancer. Moreover, systematic exploration of SDPR in gene location, species conservation, function, and potential regulatory network was illustrated in lung cancer, especially in $K R A S$-mutant tumors. In addition, our research originally unfolded the correlation between SDPR, immune checkpoint molecules, and abundance of immune infiltrations. In summary, SDPR could be a promising prognostic factor and potential target for the treatment of lung cancer, especially for $K R A S$-mutant adenocarcinomas.

\section{Supplementary Information}

The online version contains supplementary material available at https://doi. org/10.1186/s12935-021-01756-8.

Additional file 1. Additional materials, mehtods and figure 


\begin{abstract}
Abbreviations
SDPR: Serum Deprivation Protein Response; NSCLC: Non-small cell lung cancer; IHC: Immunohistochemistry; TCGA: The Cancer Genome Atlas; TFs: Transcription factors; TNFRSF18: Tumor Necrosis Factor Receptor Superfamily Member 18; TNFRSF9: Tumor Necrosis Factor Receptor Superfamily Member 9; TDO2:Tryptophan 2,3-dioxygenase 2; TME: Tumor microenvironment; CNV: Copy number variation; EGFR: Epidermal growth factor receptor; ALK: Anaplastic lymphoma kinase; ATCC1: American Type Culture Collection; GEMM: Genetically engineered mouse model; GSEA: Gene set enrichment analysis; GEO: Gene expression omnibus; DEG: Differentially expressed gene.
\end{abstract}

\section{Acknowledgements}

The authors thank the teachers of department of Radiation Oncology of Nanfang Hospital for suggestions. The authors thank technique surpport (https:// www.biowolf.cn/) for bioinformatics analysis and LetPub (www.letpub.com) for its linguistic assistance during the preparation of this manuscript.

\section{Authors' contributions}

Study design: WW, SP. Data collection (provided animals, acquired and managed patients, provided facilities, etc.): XL, SP, RW, YM, SC, YW. Analysis and interpretation of data (e.g., statistical analysis, biostatistics, computational analysis): SP, XL, WW. Writing, review, and/or revision of the manuscript: $\mathrm{XL}, \mathrm{SP}$, WW. Literature search and organization of figure and table: (i.e., reporting or organizing data, constructing databases): SP, XL, SD, QZ. Study supervision: WW. All authors read and approved the final manuscript.

\section{Funding}

This work was supported by the National Natural Science Foundation of China Grant (81172243 and 81572966 to W. Wang) and Natural Science Foundation of Guangdong Province (2017A030313883 to W. Wang).

\section{Availability of data and materials}

All data generated or analyzed during this study are included in this published article.

\section{Ethics approval and consent to participate}

The research presented here has been performed in accordance with the Declaration of Helsinki and has been approved by the ethics committee of Nanfang hospital, Southern Medical University, China.

\section{Consent for publication}

All of the authors of this article have participated in the planning and drafting and all of the authors listed have read and approved the final version including details and images. Written informed consent for the publication has been obtained from all of the authors.

\section{Competing interests}

The authors declare that they have no competing interests.

Received: 25 August 2020 Accepted: 4 January 2021

Published online: 12 January 2021

\section{References}

1. Siegel RL, Miller KD, Jemal A. Cancer statistics, 2020. Cancer J Clin. 2020;70(1):7-30.

2. Adderley $H$, Blackhall FH, Lindsay CR. KRAS-mutant non-small cell lung cancer: converging small molecules and immune checkpoint inhibition. EBioMedicine. 2019;41:711-6.

3. Tomasini P, Walia P, Labbe C, Jao K, LeighI NB. Targeting the KRAS pathway in non-small cell lung cancer. Oncologist. 2016;21(12):1450.

4. Ferrer I, Zugazagoitia J, Herbertz S, John W, Paz-Ares L, Schmid-Bindert G. KRAS-Mutant non-small cell lung cancer: from biology to therapy. Lung cancer. 2018;124:53-64.

5. Clinical Lung Cancer Genome Project (CLCGP), Network Genomic Medicine (NGM). A genomics-based classification of human lung tumors. Sci Transl Med. 2013;5(209):209ra153.

6. Cancer Genome Atlas Research Network. Comprehensive genomic characterization of squamous cell lung cancers. Nature. 2012;489(7417):519.
7. Lindsay CR, Jamal-Hanjani M, Forster M. Blackhall F. KRAS: Reasons for optimism in lung cancer. European journal of cancer; 2018. p. 99.

8. Yoshizawa A, Sumiyoshi S, Sonobe M, Kobayashi M, Fujimoto M, Kawakami F, et al. Validation of the IASLC/ATS/ERS lung adenocarcinoma classification for prognosis and association with EGFR and KRAS gene mutations: analysis of 440 Japanese patients. J Thorac Oncol. 2013;8(1):52-61.

9. ElOsta B, Behera M, Kim S, Berry LD, Sica G, Pillai RN, et al. Characteristics and outcomes of patients with metastatic KRAS-mutant lung adenocarcinomas: the lung cancer mutation consortium experience. J Thorac Oncol. 2019;14(5):876-89.

10. Janne PA, Yang JC, Kim DW, Planchard D, Ohe Y, Ramalingam SS, et al. AZD9291 in EGFR inhibitor-resistant non-small-cell lung cancer. N Engl J Med. 2015:372(18):1689-99.

11. Thai AA, Solomon BJ. Treatment of ALK-positive nonsmall cell lung cancer: recent advances. Curr Opin Oncol. 2018;30(2):84-91.

12. Loh Z, Mitchell P, John T, Arulananda S. RET-rearranged nonsmall-cell lung cancer and therapeutic implications. Intern Med J. 2019;49(12):1541-5.

13. Scheffzek K, Ahmadian MR, Kabsch W, Wiesmuller L, Lautwein A, Schmitz F, et al. The Ras-RasGAP complex: structural basis for GTPase activation and its loss in oncogenic Ras mutants. Science. 1997;277(5324):333-9.

14. Scheffzek K, Lautwein A, Kabsch W, Ahmadian MR, Wittinghofer A. Crystal structure of the GTPase-activating domain of human p120GAP and implications for the interaction with Ras. Nature. 1996;384(6609):591-6.

15. Downward J. Targeting RAS signalling pathways in cancer therapy. Nat Rev Cancer. 2003;3(1):11-22.

16. Cox AD, Fesik SW, Kimmelman AC, Luo J, Der CJ. Drugging the undruggable RAS: mission possible? Nat Rev Drug Discov. 2014;13(11):828-51.

17. Dogan S, Shen R, Ang DC, Johnson ML, D'Angelo SP, Paik PK, et al. Molecular epidemiology of EGFR and KRAS mutations in 3,026 lung adenocarcinomas: higher susceptibility of women to smoking-related KRAS-mutant cancers. Clin Cancer Res. 2012;18(22):6169-77.

18. Canon J, Rex K, Saiki AY, Mohr C, Cooke K, Bagal D, et al. The clinical KRAS (G12C) inhibitor AMG 510 drives anti-tumour immunity. Nature. 2019;575(7781):217-23.

19. Ryan MB, Fece de la Cruz F. Vertical pathway inhibition overcomes adaptive feedback resistance to KRAS(G12C) inhibition. Clin Cancer Res. 2020;26(7):1633-43.

20. Janes MR, Zhang J, Li LS, Hansen R, Peters U, Guo X, et al. Targeting KRAS mutant cancers with a covalent G12C-specific inhibitor. Cell. 2018;172(3):578-89.

21. Dong ZY, Zhong WZ, Zhang XC, Su J, Xie Z, Liu SY, et al. Potential predictive value of TP53 and KRAS mutation status for response to PD-1 blockade immunotherapy in lung adenocarcinoma. Clin Cancer Res. 2017:23(12):3012-24.

22. Liu C, Zheng S, Jin R, Wang X, Wang F, Zang R, et al. The superior efficacy of anti-PD-1/PD-L1 immunotherapy in KRAS-mutant non-small cell lung cancer that correlates with an inflammatory phenotype and increased immunogenicity. Cancer Lett. 2020;470:95-105.

23. Falk AT, Yazbeck N, Guibert N, Chamorey E, Paquet A, Ribeyre L, et al. Effect of mutant variants of the KRAS gene on PD-L1 expression and on the immune microenvironment and association with clinical outcome in lung adenocarcinoma patients. Lung Cancer. 2018;121:70-5.

24. Skoulidis F, Goldberg ME. STK11/LKB1 mutations and PD-1 inhibitor resistance in KRAS-mutant lung adenocarcinoma. Cancer Discov. 2018;8(7):822-35.

25. Hansen CG, Bright NA, Howard G, Nichols BJ. SDPR induces membrane curvature and functions in the formation of caveolae. Nat Cell Biol. 2009;11(7):807-14.

26. Wang Y, Song Z, Leng P, Liu Y. A systematic analysis reveals gene expression alteration of serum deprivation response (SDPR) gene is significantly associated with the survival of patients with cancer. Oncol Rep. 2019:42(3):1161-72.

27. Luo Y, Wu J, Wu Q, Li X, Wu J, Zhang J, et al. miR-577 regulates TGF-beta induced cancer progression through a SDPR-modulated positivefeedback loop with ERK-NF-kappaB in gastric cancer. Mol Ther. 2019;27(6):1166-82

28. Jing W, Luo P, Zhu M, Ai Q, Chai H, Tu J. Prognostic and diagnostic significance of SDPR-Cavin-2 in hepatocellular carcinoma. Cell Physiol Biochem. 2016;39(3):950-60. 
29. Wang QX, Chen ED, Cai YF, Zhou YL, Dong SY, Zhang XH, et al. Serum deprivation response functions as a tumor suppressor gene in papillary thyroid cancer. Clin Genet. 2019;96(5):418-28.

30. Wang R, Peng S, Zhang X, Wu Z, Duan H, Yuan Y. Inhibition of NF-kB improves sensitivity to irradiation and EGFR-TKIs and decreases irradiation-induced lung toxicity. Int J Cancer. 2019;144(1):200-9.

31. Liu T, Sun Q, Li Q, Yang H, Zhang Y, Wang R, et al. Dual PI3K/mTOR inhibitors, GSK2126458 and PKI-587, suppress tumor progression and increase radiosensitivity in nasopharyngeal carcinoma. Mol Cancer Ther 2015;14(2):429-39.

32. DuPage M, Dooley AL, Jacks T. Conditional mouse lung cancer models using adenoviral or lentiviral delivery of Cre recombinase. Nat Protoc. 2009:4(7):1064.

33. Yano S, Wang W, Li Q, Matsumoto K, Sakurama H, Nakamura T, et al. Hepatocyte growth factor induces gefitinib resistance of lung adenocarcinoma with epidermal growth factor receptor-activating mutations. Cancer Res. 2008;68(22):9479-87.

34. Peng $S$, Wang $R$, Zhang $X$, Ma Y, Zhong L, Li K, et al. EGFR-TKI resistance promotes immune escape in lung cancer via increased PD-L1 expression. Mol Cancer. 2019:18(1):165.

35. Gupta R, Toufaily C, Annabi B. Caveolin and cavin family members: dual roles in cancer. Biochimie. 2014;107:188-202.

36. Ozturk S, Papageorgis P, Wong CK, Lambert AW, Abdolmaleky HM, Thiagalingam A, et al. SDPR functions as a metastasis suppressor in breast cancer by promoting apoptosis. Proc Natl Acad Sci USA. 2016;113(3):638-43.

37. Yu HA, Sima CS, Shen R, Kass S, Gainor J, Shaw A, et al. Prognostic impact of KRAS mutation subtypes in 677 patients with metastatic lung adenocarcinomas. J Thorac Oncol. 2015:10(3):431-7.
38. Ihle NT, Byers LA, Kim ES, Saintigny P, Lee JJ, Blumenschein GR, et al. Effect of KRAS oncogene substitutions on protein behavior: implications for signaling and clinical outcome. J Natl Cancer Inst. 2012;104(3):228-39.

39. Shepherd FA, Domerg C, Hainaut P, Janne PA, Pignon JP, Graziano S, et al. Pooled analysis of the prognostic and predictive effects of KRAS mutation status and KRAS mutation subtype in early-stage resected non-smallcell lung cancer in four trials of adjuvant chemotherapy. J Clin Oncol. 2013;31(17):2173

40. Skoulidis F, Byers LA, Diao L, Papadimitrakopoulou VA, Tong P, Izzo J, et al. Co-occurring genomic alterations define major subsets of KRAS-mutant lung adenocarcinoma with distinct biology, immune profiles, and therapeutic vulnerabilities. Cancer Discov. 2015;5(8):860-77.

41. Arbour KC, Jordan E, Kim HR, Dienstag J, Yu HA, Sanchez-Vega F, et al. Effects of co-occurring genomic alterations on outcomes in patients with KRAS-mutant non-small cell lung cancer. Clin Cancer Res. 2018;24(2):34-40.

42. Schabath MB, Welsh EA, Fulp WJ, Chen L, Teer JK, Thompson ZJ, et al. Differential association of STK11 and TP53 with KRAS mutation-associated gene expression, proliferation and immune surveillance in lung adenocarcinoma. Oncogene. 2016;35(24):3209-16.

\section{Publisher's Note}

Springer Nature remains neutral with regard to jurisdictional claims in published maps and institutional affiliations.

Ready to submit your research? Choose BMC and benefit from:

- fast, convenient online submission

- thorough peer review by experienced researchers in your field

- rapid publication on acceptance

- support for research data, including large and complex data types

- gold Open Access which fosters wider collaboration and increased citations

- maximum visibility for your research: over $100 \mathrm{M}$ website views per year

At BMC, research is always in progress.

Learn more biomedcentral.com/submissions 